\title{
THE STUDY AND MAPPING OF THE GEOSYSTEMS TRANSFORMATION (Methodological and Methodic Aspects)
}

\author{
Tatyana Konovalova ${ }^{12}$, Alexander Sizykh ${ }^{3 *}$ \\ ${ }^{1}$ Irkutsk State University, 664033, Irkutsk, 126 Ulan-Batorskay str., Russia; \\ ${ }^{2} V$. B. Sochava Institute of Geography SB RAS, 664033, 1 Ulan-Batorskay str., Irkutsk, Russia; \\ ${ }^{3 *}$ Siberian Institute of Plant Physiology and Biochemistry SB RAS, 664033, 132 Lermontova str., Irkutsk, Russia; \\ *Corresponding Author Alexander Sizykh, e-mail: alexander.sizykh@gmail.com;
}

Received June 2021; Accepted July 2021; Published September 2021;

DOI: https://doi.org/10.31407/ijees11.422

\begin{abstract}
Mapping of geosystems attracts attention as a concise way to organize a significant amount of geographical information. Initially, the development of theoretical provisions and the compilation of geosystem maps were based on geobotanical principles and methods of integrating facies according to structural and structural-dynamic indicators. At the present stage, the task of mapping geosystems is largely determined by the need for predictive studies of their natural and anthropogenic transformations. The relevance of the development of geosystem mapping in the direction described here is determined by the need to create medium-scale maps of the transformation of geosystems, which are characterized by significant spatial and temporal scales, heterogeneous genesis, various stages of development. The creation of such maps requires the synthesis of time and space in a single whole, the comparison of the current state of the geosystem with the natural rhythms and laws of the development of the natural environment. The mapping methodology is based on the modern idea of the transformation of geosystems and the further development of the theory of geosystems by V. B. Sochava, includes techniques for displaying patterns of development and transformation of geosystem structures. Maps of the transformation of geosystems are a theoretical model of reality, synthesize information about the transformative dynamics and evolution of geosystems, the direction of their rearrangements, both in natural and anthropogenic conditions. The article presents a scheme of transformation of geosystems and methods of its mapping.
\end{abstract}

Key words: geosystem, structure, evolutionary and dynamic transformations, transformation factors, map legend. 